Pak. j. sci. ind. res. Ser. A: phys. sci. 201255 (2) 59-67

\title{
Experimental and Theoretical Ultrasonic Velocities of Binary Liquid Mixtures at Temperatures $(303.15,308.15,313.15$ and 318.15)K - Comparative Study
}

\author{
Kolla Narendra*a ${ }^{* a}$, Chintalapati Srinivasu ${ }^{b}$ and Piratla Narayanamurthy ${ }^{c}$ \\ ${ }^{a}$ Department of Physics, V.R.Siddhartha Engineering College, Kanuru, Vijayawada-520007, \\ Andhrapradesh, India \\ ${ }^{\mathbf{b}}$ Department of Physics, Andhra Loyola College(Autonomous), Vijayawada, A.P., India \\ ${ }^{a}$ Department of Physics, Acharya Nagarjuna University, Nagarjuna Nagar, Guntur, India
}

(received April 12, 2011; revised June 28, 2011; accepted July 5, 2011)

\begin{abstract}
Ultrasonic velocities of binary liquid mixtures of anisaldehyde with $o$-xylene, $m$-xylene and $p$-xylene were measured using ultrasonic interferometer at 303.15, 308.15, 313.15 and $318.15 \mathrm{~K}$ over the entire composition range. A comparative study of Nomoto, Van Dael-Vangeel, Impedance dependence, Rao's specific velocity and Junjie's relations for predicting the ultrasonic velocities of a liquid was carried out to test their validity for the binary systems over the entire mole fraction range at the four temperatures. A good agreement was found between experimental and Nomoto's ultrasonic velocities followed by Rao's specific velocity. $\mathrm{U}_{\text {exp }}^{2} / \mathrm{U}^{2}$ imx was also evaluated for non-ideality in the mixtures. The results are explained in terms of molecular interactions occurring in these binary liquid mixtures.
\end{abstract}

Keywords: ultrasonic velocities, anisaldehyde, xylenes, binary mixtures, theoretical models

\section{Introduction}

In estimating the nature of molecular interactions and to know the physico-chemical behaviour, measurement of ultrasonic velocities in liquids and liquid mixtures has gained much importance. Several researchers (Narendra et al., 2010; Uvarani and Punitha, 2009; Rao et al., 2005; Ali et al., 2001; Arumugam et al., 1998) carried out ultrasonic investigations on binary liquid mixtures and compared the experimental results with the theoretical relations (Nomoto, 1958) of Van Dael and Vangeel (Bhatia et al., 2010), Impedance dependence (Baluja and Parsania, 1995), Rao's specific velocity (Kumar and Rao, 2007) and Junjie's (Azhagiri et al., 2009) equations and the results are explained in terms of molecular interactions. Densities and ultrasonic velocities in three binary liquid mixtures are measured experimentally and the experimental values of velocities are compared with the above mentioned theoretical relations at four temperatures. Based on the deviation in $\mathrm{U}_{\text {exp }}^{2} / \mathrm{U}^{2}{ }_{\mathrm{imx}}$ the molecular interactions are studied.

*Author for correspondence; E-mail: narenk75@gmail.com

\section{Materials and Methods}

The chemicals used were of AnalaR grade and obtained from SRL Chemicals (anisaldehyde) and Merck (xylenes). The chemicals were purified by standard procedure (Perrin and Armarego, 1980). The purity of samples was checked by comparing experimental values of density and ultrasonic velocity with the available literature (Baskaran and Kubendran, 2008; Al-Kandary et al., 2006). Job's method of continuous variation was used to prepare the mixtures of required proportions. The prepared mixtures were preserved in well-stoppard conical flasks. After mixing the liquids thoroughly, the flasks were left undisturbed to allow them to attain thermal equilibrium.

The densities of pure liquids and liquid mixtures were measured using a specific gravity bottle with an accuracy of $\pm 0.5 \%$. Weights were measured with an electronic balance (Shimadzu AUY220, Japan) capable of measuring up to $0.1 \mathrm{mg}$. An average of 4-5 measurements was taken for each sample.

The ultrasonic velocities were measured using single crystal ultrasonic pulse echo interferometer (Mittal enterprises, India; Model: F-80X). It consists of a high 
frequency generator and a measuring cell. The measurements of ultrasonic velocities were made at the fixed frequency of $3 \mathrm{MHz}$. The calibration of the equipment was done by measuring the velocity in carbon tetrachloride and benzene. The results were in good agreement with the literature values (Lide, 1995). The ultrasonic velocity had an accuracy of $\pm 0.5 \mathrm{~m} / \mathrm{s}$. The temperature was controlled by circulating water around the liquid cell from thermostatically controlled constant temperature water bath.

\section{Results and Discussion}

Comparison of the experimental values with the earlier reported values of density, for checking the purity of samples, is given in Table 1. Theoretical values of ultrasonic velocities in the present binary liquid mixtures were calculated using different theories and empirical relations. Comparison of theoretical values of ultrasonic velocities with those obtained experimentally in the present binary liquid mixtures is expected to reveal the nature of interaction between the component molecules in the mixture. Such theoretical study is useful in building the comprehensive theoretical model for the liquid mixtures. Theoretical values of ultrasonic velocities in the mixtures: anisaldehyde $+o$-xylene, + $m$-xylene and $+p$-xylene at different mole fractions of anisaldehyde for different temperatures were calculated using the following theories and relations:

Nomoto (1958) established an empirical relation for ultrasonic velocity in binary liquid mixtures as:

$$
\mathrm{U}_{\mathrm{N}}=\left[\left(\mathrm{x}_{1} \mathrm{R}_{1}+\mathrm{x}_{2} \mathrm{R}_{2}\right) /\left(\mathrm{x}_{1} \mathrm{~V}_{1}+\mathrm{x}_{2} \mathrm{~V}_{2}\right)\right]^{3}
$$

Where, $\mathrm{R}$ is molar sound velocity, $\mathrm{x}_{1}$ and $\mathrm{x}_{2}$ are the mole fractions of the $1^{\text {st }}$ and the $2^{\text {nd }}$ components of the liquid mixture and $\mathrm{V}$ is molar volume.

Table 1. Experimental and literature values of density and ultrasonic velocity of pure liquids at $303.15 \mathrm{~K}$.

\begin{tabular}{lllll}
\hline \hline Pure liquid & \multicolumn{2}{l}{$\rho .10^{-3} /\left(\mathrm{kg} / \mathrm{m}^{3}\right)$} & $\mathrm{u} /(\mathrm{m} / \mathrm{s})$ \\
\cline { 2 - 5 } & Exp. & Lit. & Exp. & Lit. \\
\hline Anisaldehyde & 1.1252 & $1.1255^{\mathrm{a}}$ & 1694 & $1684^{\mathrm{a}}$ \\
o-xylene & 0.8707 & $0.8707^{\mathrm{b}}$ & 1339 & $1328.30^{\mathrm{b}}$ \\
$m$-xylene & 0.8557 & $0.8555^{\mathrm{b}}$ & 1304 & $1300.34^{\mathrm{b}}$ \\
$p$-xylene & 0.8528 & $0.8523^{\mathrm{b}}$ & 1288 & $1289.68^{\mathrm{b}}$ \\
\hline \hline
\end{tabular}

$\overline{{ }^{a} \text { Baskaran and Kubendran (2008); }{ }^{\mathrm{b}} \text { Al-Kandary et al. (2006). }}$
Van Deal and Vangeel ideal mixing relation is:

$$
\mathrm{U}_{\mathrm{imx}}=\left[\left(\mathrm{x}_{1} / \mathrm{M}_{1} \mathrm{U}^{2}{ }_{1}+\mathrm{x}_{2} / \mathrm{M}_{2} \mathrm{U}^{2}\right)\left(\mathrm{x}_{1} \mathrm{M}_{1}+\mathrm{x}_{2} \mathrm{M}_{2}\right)\right]^{-1 / 2}(2)
$$

Where, $\mathrm{U}_{\mathrm{imx}}$ is the ideal mixing ultrasonic velocity in liquid mixture. $\mathrm{U}_{1}$ and $\mathrm{U}_{2}$ are ultrasonic velocities of individual compounds.

Impedance dependent relation:

$$
\mathrm{U}_{\mathrm{Im}}=\Sigma \mathrm{x}_{\mathrm{i}} \mathrm{Z}_{\mathrm{i}} / \sum \mathrm{x}_{\mathrm{i}} \rho_{\mathrm{i}}
$$

Where, $x_{i}$ is the mole fraction, $\rho_{i}$ the density of the mixture and $\mathrm{Z}_{\mathrm{i}}$ is the acoustic impedance.

Rao's specific velocity:

$$
\mathrm{U}_{\mathrm{R}}=\left(\sum \mathrm{x}_{\mathrm{i}} \mathrm{r}_{\mathrm{i}} \rho_{\mathrm{i}}\right)^{3}
$$

Where, $x_{i}$ is the mole fraction, $U_{i}$ is the ultrasonic velocity, $\rho_{i}$ the density of the mixture and $r_{i}$ is the Rao's specific sound velocity $=\mathrm{U}^{1 / 3}{ }_{\mathrm{i}} / \rho_{\mathrm{i}}$

Junjie's equation:

$$
\begin{aligned}
\mathrm{U}_{\mathrm{J}}= & \left(\mathrm{x}_{1} \mathrm{M}_{1 /} / \rho_{1}+\mathrm{x}_{2} \mathrm{M}_{2} / \rho_{2}\right) /\left[\left\{\mathrm{x}_{1} \mathrm{M}_{1}+\mathrm{x}_{2} \mathrm{M}_{2}\right\}^{1 / 2} \times\left\{\mathrm{x}_{1} \mathrm{M}_{1} / \rho_{1}\right.\right. \\
& \left.\left.\mathrm{U}_{1}^{2}+\mathrm{x}_{2} \mathrm{M}_{2} / \rho_{2} \mathrm{U}_{2}^{2}\right\}\right]^{1 / 2}
\end{aligned}
$$

Where, $\mathrm{M}_{1}, \mathrm{M}_{2}$ are molecular weights of constituent components, $\rho_{1}$ and $\rho_{2}$ are the densities of constituent components.

The theoretical evaluation of sound velocity based on different models in liquid mixtures has been used to correlate with the experimental findings. The theoretical values of ultrasonic velocities calculated using the equations 1-5 along with the experimental values for all the three mixtures at temperatures of 303.15, 308.15, 313.15 and $318.15 \mathrm{~K}$ are given in Tables $2-4$. The validity of these theories was checked by percentage deviation for all the mixtures at all the temperatures and is given in Tables 5-7.

It can be seen from Tables 2-4 that the theoretical values of ultrasonic velocity calculated using various theories show deviation from the experimental values. The limitations and approximation incorporated in these theories are responsible for the deviations of the theoretical values from the experimental values. In Nomoto's theory, it is supposed that the volume does not change on mixing. But on mixing two liquids, the interaction between the molecules of the two liquids takes place because of the presence of various types of forces such as dispersive forces, charge transfer, hydrogen bonding, dipole-dipole and dipole-induced dipole interactions. Thus, the observed deviation of 
Table 2. Experimental and theoretical values of velocities $(\mathrm{m} / \mathrm{s})$ in anisaldehyde $+o$-xylene system at different temperatures

\begin{tabular}{|c|c|c|c|c|c|c|}
\hline $\mathrm{x}_{1}$ & $\mathrm{U}_{\exp }$ & $\mathrm{U}_{\mathrm{N}}$ & $\mathrm{U}_{\mathrm{imx}}$ & $\mathrm{U}_{\mathrm{Im}}$ & $\mathrm{U}_{\mathrm{R}}$ & $\mathrm{U}_{\mathrm{J}}$ \\
\hline \multicolumn{7}{|c|}{$303.15 \mathrm{~K}$} \\
\hline 0.0000 & 1339 & 1338.75 & 1338.75 & 1338.75 & 1338.75 & 1429.23 \\
\hline 0.1007 & 1382 & 1371.83 & 1367.64 & 1383.69 & 1372.06 & 1437.51 \\
\hline 0.2012 & 1415 & 1405.44 & 1397.76 & 1426.08 & 1405.85 & 1447.47 \\
\hline 0.3016 & 1451 & 1439.60 & 1429.19 & 1466.11 & 1440.15 & 1459.18 \\
\hline 0.4018 & 1485 & 1474.31 & 1462.01 & 1503.99 & 1474.95 & 1472.77 \\
\hline 0.5019 & 1520 & 1509.57 & 1496.33 & 1539.88 & 1510.25 & 1488.36 \\
\hline 0.6018 & 1555 & 1545.39 & 1532.25 & 1573.93 & 1546.05 & 1506.11 \\
\hline 0.7016 & 1596 & 1581.77 & 1569.87 & 1606.28 & 1582.36 & 1526.22 \\
\hline 0.8012 & 1650 & 1618.72 & 1609.33 & 1637.06 & 1619.17 & 1548.93 \\
\hline 0.9007 & 1673 & 1656.24 & 1650.77 & 1666.37 & 1656.50 & 1574.53 \\
\hline 1.0000 & 1694 & 1694.33 & 1694.33 & 1694.33 & 1694.33 & 1603.38 \\
\hline \multicolumn{7}{|c|}{$308.15 \mathrm{~K}$} \\
\hline 0.0000 & 1315 & 1315.00 & 1315.00 & 1315.00 & 1315.00 & 1410.31 \\
\hline 0.1007 & 1357 & 1348.36 & 1343.80 & 1359.54 & 1348.27 & 1418.40 \\
\hline 0.2012 & 1390 & 1382.21 & 1373.84 & 1401.65 & 1382.05 & 1428.18 \\
\hline 0.3016 & 1425 & 1416.55 & 1405.21 & 1441.52 & 1416.33 & 1439.75 \\
\hline 0.4018 & 1460 & 1451.38 & 1438.00 & 1479.34 & 1451.12 & 1453.23 \\
\hline 0.5019 & 1495 & 1486.70 & 1472.30 & 1515.24 & 1486.42 & 1468.75 \\
\hline 0.6018 & 1530 & 1522.50 & 1508.22 & 1549.39 & 1522.24 & 1486.49 \\
\hline 0.7016 & 1570 & 1558.80 & 1545.88 & 1581.89 & 1558.57 & 1506.66 \\
\hline 0.8012 & 1620 & 1595.60 & 1585.42 & 1612.87 & 1595.41 & 1529.52 \\
\hline 0.9007 & 1647 & 1632.89 & 1626.96 & 1642.43 & 1632.78 & 1555.37 \\
\hline 1.0000 & 1671 & 1670.67 & 1670.67 & 1670.67 & 1670.67 & 1584.59 \\
\hline \multicolumn{7}{|c|}{$313.15 \mathrm{~K}$} \\
\hline 0.0000 & 1297 & 1297.50 & 1297.50 & 1297.50 & 1297.50 & 1392.91 \\
\hline 0.1007 & 1335 & 1328.92 & 1324.78 & 1339.31 & 1328.83 & 1399.93 \\
\hline 0.2012 & 1367 & 1360.77 & 1353.18 & 1378.83 & 1360.61 & 1408.53 \\
\hline 0.3016 & 1400 & 1393.05 & 1382.80 & 1416.26 & 1392.85 & 1418.80 \\
\hline 0.4018 & 1433 & 1425.78 & 1413.69 & 1451.75 & 1425.54 & 1430.83 \\
\hline 0.5019 & 1466 & 1458.94 & 1445.95 & 1485.45 & 1458.69 & 1444.74 \\
\hline 0.6018 & 1499 & 1492.54 & 1479.67 & 1517.50 & 1492.29 & 1460.67 \\
\hline 0.7016 & 1535 & 1526.57 & 1514.95 & 1548.00 & 1526.36 & 1478.80 \\
\hline 0.8012 & 1581 & 1561.05 & 1551.90 & 1577.08 & 1560.89 & 1499.34 \\
\hline 0.9007 & 1608 & 1595.97 & 1590.65 & 1604.83 & 1595.88 & 1522.55 \\
\hline 1.0000 & 1631 & 1631.33 & 1631.33 & 1631.33 & 1631.33 & 1548.74 \\
\hline \multicolumn{7}{|c|}{$318.15 \mathrm{~K}$} \\
\hline 0.0000 & 1278 & 1278.15 & 1278.15 & 1278.15 & 1278.15 & 1373.56 \\
\hline 0.1007 & 1309 & 1304.70 & 1301.49 & 1313.00 & 1304.58 & 1377.71 \\
\hline 0.2012 & 1336 & 1331.56 & 1325.68 & 1345.97 & 1331.33 & 1383.17 \\
\hline 0.3016 & 1363 & 1358.71 & 1350.79 & 1377.20 & 1358.40 & 1389.97 \\
\hline 0.4018 & 1391 & 1386.15 & 1376.85 & 1406.83 & 1385.80 & 1398.18 \\
\hline 0.5019 & 1419 & 1413.89 & 1403.93 & 1434.98 & 1413.52 & 1407.84 \\
\hline 0.6018 & 1446 & 1441.92 & 1432.09 & 1461.76 & 1441.56 & 1419.04 \\
\hline 0.7016 & 1476 & 1470.25 & 1461.40 & 1487.27 & 1469.93 & 1431.86 \\
\hline 0.8012 & 1515 & 1498.87 & 1491.93 & 1511.59 & 1498.63 & 1446.43 \\
\hline 0.9007 & 1536 & 1527.79 & 1523.77 & 1534.80 & 1527.65 & 1462.88 \\
\hline 1.0000 & 1557 & 1557.00 & 1557.00 & 1556.98 & 1557.00 & 1481.38 \\
\hline
\end{tabular}


Table 3. Experimental and theoretical values of velocities $(\mathrm{m} / \mathrm{s})$ in anisaldehyde $+m$-xylene system at different temperatures

\begin{tabular}{|c|c|c|c|c|c|c|}
\hline $\mathrm{x}_{1}$ & $\mathrm{U}_{\exp }$ & $\mathrm{U}_{\mathrm{N}}$ & $\mathrm{U}_{\mathrm{imx}}$ & $\mathrm{U}_{\mathrm{Im}}$ & $\mathrm{U}_{\mathrm{R}}$ & $\mathrm{U}_{\mathrm{J}}$ \\
\hline \multicolumn{7}{|c|}{$303.15 \mathrm{~K}$} \\
\hline 0.0000 & 1304 & 1304.21 & 1304.21 & 1304.21 & 1304.21 & 1409.90 \\
\hline 0.1023 & 1354 & 1340.20 & 1335.65 & 1355.03 & 1341.02 & 1417.38 \\
\hline 0.2040 & 1391 & 1376.84 & 1368.48 & 1402.55 & 1378.33 & 1426.86 \\
\hline 0.3053 & 1430 & 1414.15 & 1402.79 & 1447.07 & 1416.13 & 1438.45 \\
\hline 0.4060 & 1467 & 1452.12 & 1438.68 & 1488.87 & 1454.43 & 1452.30 \\
\hline 0.5062 & 1505 & 1490.77 & 1476.26 & 1528.19 & 1493.21 & 1468.60 \\
\hline 0.6060 & 1543 & 1530.10 & 1515.66 & 1565.25 & 1532.48 & 1487.59 \\
\hline 0.7052 & 1589 & 1570.12 & 1557.01 & 1600.23 & 1572.22 & 1509.54 \\
\hline 0.8040 & 1646 & 1610.82 & 1600.46 & 1633.31 & 1612.45 & 1534.82 \\
\hline 0.9022 & 1671 & 1652.23 & 1646.17 & 1664.63 & 1653.16 & 1563.88 \\
\hline 1.0000 & 1694 & 1694.33 & 1694.33 & 1694.33 & 1694.33 & 1597.29 \\
\hline \multicolumn{7}{|c|}{$308.15 \mathrm{~K}$} \\
\hline 0.0000 & 1285 & 1285.26 & 1285.27 & 1285.27 & 1285.26 & 1395.12 \\
\hline 0.1023 & 1332 & 1320.94 & 1316.32 & 1335.31 & 1321.63 & 1402.96 \\
\hline 0.2040 & 1369 & 1357.24 & 1348.73 & 1382.14 & 1358.48 & 1412.77 \\
\hline 0.3053 & 1407 & 1394.17 & 1382.61 & 1426.06 & 1395.82 & 1424.66 \\
\hline 0.4060 & 1446 & 1431.73 & 1418.06 & 1467.32 & 1433.65 & 1438.78 \\
\hline 0.5062 & 1483 & 1469.94 & 1455.18 & 1506.18 & 1471.96 & 1455.32 \\
\hline 0.6060 & 1520 & 1508.78 & 1494.10 & 1542.82 & 1510.75 & 1474.51 \\
\hline 0.7052 & 1566 & 1548.27 & 1534.96 & 1577.43 & 1550.02 & 1496.63 \\
\hline 0.8040 & 1618 & 1588.42 & 1577.89 & 1610.19 & 1589.77 & 1522.04 \\
\hline 0.9022 & 1647 & 1629.21 & 1623.07 & 1641.22 & 1629.98 & 1551.17 \\
\hline 1.0000 & 1671 & 1670.67 & 1670.67 & 1670.67 & 1670.67 & 1584.57 \\
\hline \multicolumn{7}{|c|}{$313.15 \mathrm{~K}$} \\
\hline 0.0000 & 1266 & 1266.32 & 1266.32 & 1266.32 & 1266.32 & 1376.92 \\
\hline 0.1023 & 1309 & 1300.16 & 1295.98 & 1313.77 & 1300.86 & 1383.74 \\
\hline 0.2040 & 1345 & 1334.57 & 1326.89 & 1358.17 & 1335.84 & 1392.42 \\
\hline 0.3053 & 1381 & 1369.57 & 1359.15 & 1399.78 & 1371.26 & 1403.08 \\
\hline 0.4060 & 1417 & 1405.17 & 1392.85 & 1438.88 & 1407.12 & 1415.82 \\
\hline 0.5062 & 1453 & 1441.35 & 1428.08 & 1475.67 & 1443.41 & 1430.83 \\
\hline 0.6060 & 1488 & 1523.75 & 1464.95 & 1510.36 & 1480.14 & 1448.30 \\
\hline 0.7052 & 1530 & 1550.41 & 1503.57 & 1543.12 & 1517.30 & 1468.48 \\
\hline 0.8040 & 1577 & 1577.23 & 1544.08 & 1574.12 & 1554.89 & 1491.68 \\
\hline 0.9022 & 1607 & 1604.20 & 1586.61 & 1603.48 & 1592.90 & 1518.27 \\
\hline 1.0000 & 1631 & 1631.33 & 1631.33 & 1631.33 & 1631.33 & 1548.74 \\
\hline \multicolumn{7}{|c|}{$318.15 \mathrm{~K}$} \\
\hline 0.0000 & 1244 & 1244.21 & 1244.21 & 1244.21 & 1244.21 & 1357.14 \\
\hline 0.1023 & 1280 & 1273.42 & 1270.28 & 1284.95 & 1274.08 & 1361.13 \\
\hline 0.2040 & 1311 & 1303.08 & 1297.33 & 1323.03 & 1304.26 & 1366.72 \\
\hline 0.3053 & 1341 & 1333.19 & 1325.42 & 1358.72 & 1334.77 & 1373.98 \\
\hline 0.4060 & 1372 & 1363.77 & 1354.61 & 1392.24 & 1365.58 & 1382.99 \\
\hline 0.5062 & 1403 & 1394.80 & 1384.97 & 1423.76 & 1396.72 & 1393.84 \\
\hline 0.6060 & 1434 & 1426.30 & 1416.57 & 1453.48 & 1428.16 & 1406.66 \\
\hline 0.7052 & 1467 & 1458.27 & 1449.48 & 1481.53 & 1459.91 & 1421.62 \\
\hline 0.8040 & 1510 & 1490.70 & 1483.79 & 1508.05 & 1491.97 & 1438.89 \\
\hline 0.9022 & 1534 & 1523.61 & 1519.60 & 1533.18 & 1524.33 & 1458.71 \\
\hline 1.0000 & 1557 & 1557.00 & 1557.00 & 1557.00 & 1557.00 & 1481.38 \\
\hline
\end{tabular}


Table 4. Experimental and theoretical values of velocities $(\mathrm{m} / \mathrm{s})$ in anisaldehyde $+p$-xylene system at different temperatures

\begin{tabular}{|c|c|c|c|c|c|c|}
\hline $\mathrm{x}_{1}$ & $\mathrm{U}_{\exp }$ & $\mathrm{U}_{\mathrm{N}}$ & $\mathrm{U}_{\mathrm{imx}}$ & $\mathrm{U}_{\mathrm{Im}}$ & $\mathrm{U}_{\mathrm{R}}$ & $\mathrm{U}_{\mathrm{J}}$ \\
\hline \multicolumn{7}{|c|}{$303.15 \mathrm{~K}$} \\
\hline 0.0000 & 1288 & 1288.43 & 1288.43 & 1288.43 & 1288.43 & 1395.20 \\
\hline 0.1026 & 1342 & 1325.73 & 1320.86 & 1341.62 & 1326.70 & 1403.35 \\
\hline 0.2046 & 1380 & 1363.75 & 1354.77 & 1391.27 & 1365.50 & 1413.59 \\
\hline 0.3060 & 1420 & 1402.48 & 1390.27 & 1437.71 & 1404.82 & 1426.07 \\
\hline 0.4068 & 1459 & 1441.94 & 1427.46 & 1481.25 & 1444.66 & 1440.94 \\
\hline 0.5071 & 1499 & 1482.13 & 1466.48 & 1522.15 & 1485.01 & 1458.44 \\
\hline 0.6068 & 1538 & 1523.07 & 1507.46 & 1560.64 & 1525.87 & 1478.82 \\
\hline 0.7059 & 1586 & 1564.75 & 1550.56 & 1596.93 & 1567.24 & 1502.42 \\
\hline 0.8045 & 1645 & 1607.18 & 1595.94 & 1631.20 & 1609.11 & 1529.66 \\
\hline 0.9025 & 1671 & 1650.37 & 1643.79 & 1663.62 & 1651.47 & 1561.06 \\
\hline 1.0000 & 1694 & 1694.33 & 1694.33 & 1694.33 & 1694.33 & 1597.29 \\
\hline \multicolumn{7}{|c|}{$308.15 \mathrm{~K}$} \\
\hline 0.0000 & 1276 & 1275.79 & 1275.79 & 1275.79 & 1275.79 & 1387.06 \\
\hline 0.1026 & 1326 & 1312.27 & 1307.47 & 1327.36 & 1313.08 & 1395.21 \\
\hline 0.2046 & 1363 & 1349.40 & 1340.57 & 1375.53 & 1350.86 & 1405.39 \\
\hline 0.3060 & 1402 & 1387.19 & 1375.20 & 1420.64 & 1389.14 & 1417.73 \\
\hline 0.4068 & 1441 & 1425.65 & 1411.45 & 1462.97 & 1427.91 & 1432.40 \\
\hline 0.5071 & 1479 & 1464.78 & 1449.45 & 1502.77 & 1467.17 & 1449.59 \\
\hline 0.6068 & 1518 & 1504.59 & 1489.32 & 1540.25 & 1506.91 & 1469.55 \\
\hline 0.7059 & 1565 & 1545.08 & 1531.21 & 1575.62 & 1547.14 & 1492.59 \\
\hline 0.8045 & 1617 & 1586.25 & 1575.28 & 1609.04 & 1587.84 & 1519.10 \\
\hline 0.9025 & 1647 & 1628.11 & 1621.70 & 1640.68 & 1629.02 & 1549.56 \\
\hline 1.0000 & 1671 & 1670.67 & 1670.67 & 1670.67 & 1670.67 & 1584.59 \\
\hline \multicolumn{7}{|c|}{$313.15 \mathrm{~K}$} \\
\hline 0.0000 & 1254 & 1253.68 & 1253.68 & 1253.68 & 1253.68 & 1365.44 \\
\hline 0.1026 & 1299 & 1288.58 & 1284.17 & 1303.06 & 1289.41 & 1372.77 \\
\hline 0.2046 & 1336 & 1324.10 & 1315.99 & 1349.18 & 1325.60 & 1382.05 \\
\hline 0.3060 & 1374 & 1360.25 & 1349.23 & 1392.35 & 1362.25 & 1393.39 \\
\hline 0.4068 & 1411 & 1397.04 & 1384.00 & 1432.85 & 1399.35 & 1406.95 \\
\hline 0.5071 & 1448 & 1434.46 & 1420.39 & 1470.90 & 1436.91 & 1422.90 \\
\hline 0.6068 & 1484 & 1472.53 & 1458.53 & 1506.73 & 1474.91 & 1441.47 \\
\hline 0.7059 & 1529 & 1511.24 & 1498.54 & 1540.53 & 1513.36 & 1462.94 \\
\hline 0.8045 & 1576 & 1550.61 & 1540.57 & 1572.47 & 1552.25 & 1487.67 \\
\hline 0.9025 & 1607 & 1590.64 & 1584.78 & 1602.69 & 1591.57 & 1516.08 \\
\hline 1.0000 & 1631 & 1631.33 & 1631.33 & 1631.33 & 1631.33 & 1548.74 \\
\hline \multicolumn{7}{|c|}{$318.15 \mathrm{~K}$} \\
\hline 0.0000 & 1235 & 1234.73 & 1234.73 & 1234.74 & 1234.74 & 1349.69 \\
\hline 0.1026 & 1273 & 1264.73 & 1261.50 & 1276.99 & 1265.53 & 1353.96 \\
\hline 0.2046 & 1304 & 1295.22 & 1289.30 & 1316.41 & 1296.64 & 1359.91 \\
\hline 0.3060 & 1336 & 1326.19 & 1318.18 & 1353.29 & 1328.09 & 1367.60 \\
\hline 0.4068 & 1368 & 1357.65 & 1348.22 & 1387.86 & 1359.85 & 1377.12 \\
\hline 0.5071 & 1400 & 1389.61 & 1379.47 & 1420.33 & 1391.93 & 1388.59 \\
\hline 0.6068 & 1431 & 1422.07 & 1412.03 & 1450.89 & 1424.33 & 1402.14 \\
\hline 0.7059 & 1466 & 1455.04 & 1445.97 & 1479.69 & 1457.03 & 1417.95 \\
\hline 0.8045 & 1510 & 1488.51 & 1481.37 & 1506.90 & 1490.05 & 1436.24 \\
\hline 0.9025 & 1534 & 1522.50 & 1518.35 & 1532.63 & 1523.37 & 1457.28 \\
\hline 1.0000 & 1557 & 1557.00 & 1557.00 & 1557.00 & 1557.00 & 1481.38 \\
\hline
\end{tabular}


theoretical values of velocity from the experimental values shows that the molecular interactions are taking place (Saravanakumar et al., 2010; Rao et al., 2007; Anuradha et al., 2005; Rao et al., 2005; Ali et al., 2002) between the unlike molecules in the liquid mixture.

Anisaldehyde $+o$-xylene mixture. A close perusal of Table 5 indicates that Nomoto's relation, with minimum deviations in the range 0.60 to $1.91 \%$, predicts the experimental data well, followed by Rao's relation, with deviations in the range 0.56 to $1.88 \%$, then by impedance relation showing deviations in the range -1.31 to $0.80 \%$. However, ideal mixing relation and Junjie's theory show the maximum deviations in the range 1.02 to $2.48 \%$ and -6.76 to $6.14 \%$, respectively, over the whole mole fraction of anisaldehyde at a temperature of $303.15 \mathrm{~K}$.

Anisaldehyde $+\boldsymbol{m}$-xylene mixture. For this mixture, Table 6 reveals that percentage deviations for Nomoto's relation, Ideal mixing relation, Impedance relation, Rao's specific velocity relation and Junjie theory are respectively in the range 0.86 to $2.15 ; 1.34$ to 2.78 ; -1.54 to $0.79 ; 0.70$ to 2.05 and -8.10 to 6.77 over the whole mole fraction range of anisaldehyde at $303.15 \mathrm{~K}$. Thus, for this mixture the Nomoto's relation seems to predict the experimental data well, followed by Rao's relation at all temperatures studied.

Anisaldehyde $+\boldsymbol{p}$-xylene mixture. It can be seen from Table 7 that the percentage deviation is minimum for Nomoto's relation followed by Rao's relation in the range 1.00 to 2.29 and 0.81 to 2.17 , respectively, over the whole mole fraction range of anisaldehyde at $303.15 \mathrm{~K}$. At all the temperatures studied, the minimum deviation is observed in Nomoto's relation followed by Rao's relation.

It was also observed (Tables 5-7) that, the percentage deviations between theoretical and experimental value of ultrasonic velocities decrease with increase of temperature. It may be due to breaking of hetero - and homo-molecular clusters at higher temperatures. On increasing the temperature, the ultrasonic velocity decresases in the three binary liquid mixtures. This is probably due to the fact that the thermal energy activates the molecule, which would increase the rate of association of unlike molecules (Rastogi et al., 2002).

Plots of $\mathrm{U}^{2} \exp / \mathrm{U}^{2}$ imx against mole fraction of anisaldehyde for all the three mixtures of anisaldehyde with $o$-xylene, with $m$-xylene and with $p$-xylene are given in Fig. 1 to 3, respectively. $\mathrm{U}_{\text {exp }}^{2} / \mathrm{U}^{2}$ imx is observed
Table 5. Percentage deviation between experimental and theoretical values of velocities in anisaldehyde $+o$-xylene system at varying temperatures

\begin{tabular}{|c|c|c|c|c|c|}
\hline $\mathrm{x}_{1}$ & $\% \mathrm{U}_{\mathrm{N}}$ & $\% \mathrm{U}_{\mathrm{imx}}$ & $\% \mathrm{U}_{\mathrm{Im}}$ & $\% \mathrm{U}_{\mathrm{R}}$ & $\% \mathrm{U}_{\mathrm{J}}$ \\
\hline \multicolumn{6}{|c|}{$303.15 \mathrm{~K}$} \\
\hline 0.0000 & 0.00 & 0.00 & 0.00 & 0.00 & -6.76 \\
\hline 0.1007 & 0.72 & 1.02 & -0.14 & 0.70 & -4.04 \\
\hline 0.2012 & 0.71 & 1.25 & -0.75 & 0.68 & -2.26 \\
\hline 0.3016 & 0.77 & 1.49 & -1.06 & 0.73 & -0.58 \\
\hline 0.4018 & 0.75 & 1.58 & -1.25 & 0.71 & 0.86 \\
\hline 0.5019 & 0.69 & .56 & -1.31 & 0.64 & 2.08 \\
\hline 0.6018 & 0.60 & .45 & -1.24 & 0.56 & 3.13 \\
\hline 0.7016 & 0.92 & 1.66 & -0.62 & 0.88 & 4.40 \\
\hline 0.8012 & 1.91 & 2.48 & 0.80 & 1.88 & 6.14 \\
\hline 0.9007 & 1.03 & 1.36 & 0.42 & 1.01 & 5.91 \\
\hline 1.0000 & 0.00 & 0.00 & 0.00 & 0.00 & 5.37 \\
\hline \multicolumn{6}{|c|}{$308.15 \mathrm{~K}$} \\
\hline .0000 & 0.00 & 0.00 & 0.00 & 0.00 & -7.25 \\
\hline 0.1007 & 0.62 & 95 & -0.21 & 0.62 & -4.54 \\
\hline 0.2012 & 0.56 & 1.16 & -0.84 & 0.57 & -2.7 \\
\hline 0.3016 & 0.60 & 1.40 & -1.15 & 0.62 & -1.02 \\
\hline 0.4018 & 0.60 & 1.52 & -1.31 & 0.62 & 0.48 \\
\hline 0.5019 & 0.56 & 1.52 & -1.35 & 0.57 & 1.76 \\
\hline 0.6018 & 0.48 & 1.42 & -1.28 & 0.50 & 2.84 \\
\hline 0.7016 & 0.72 & & -0.75 & 0.74 & 4.05 \\
\hline 8012 & 1.51 & & 0.44 & 1.52 & 5.59 \\
\hline 0.900 & 0.88 & & 0.7 & 0.88 & 5.58 \\
\hline 1.0000 & 0.00 & 0.00 & 0.00 & 0.0 & 5.15 \\
\hline \multicolumn{6}{|c|}{$313.15 \mathrm{~K}$} \\
\hline .0000 & 0.00 & 0.00 & 0.00 & 0.00 & -7.35 \\
\hline 0.1007 & 0.47 & 0.78 & -0.30 & 0.48 & -4.84 \\
\hline 0.2012 & 0.47 & 1.02 & -0.85 & 0.48 & -3.03 \\
\hline 0.3016 & 0.50 & 1.24 & -1.15 & 0.52 & -1.34 \\
\hline 0.4018 & 0.49 & 1.34 & -1.32 & 0.51 & 0.14 \\
\hline 0.5019 & 0.48 & 136 & -1.33 & 0.50 & 1.45 \\
\hline 0.6018 & 0.42 & 1.28 & -1.24 & 0.44 & 2.55 \\
\hline 0.7016 & 0 & 1.30 & -0.86 & 0.55 & 3.65 \\
\hline 0.8012 & 1.26 & 1.84 & 0.2 & 1.27 & 5.16 \\
\hline 0.9007 & 0.75 & 1.08 & 0.20 & 0.76 & 5.32 \\
\hline 1.0000 & 0.00 & 0.00 & 0.00 & 0.00 & 5.06 \\
\hline \multicolumn{6}{|c|}{$318.15 \mathrm{~K}$} \\
\hline .0000 & 0.00 & U. & 0 . & 0.00 & -7.4 \\
\hline 0.1007 & 0.31 & 0.55 & -0.33 & 0.32 & -5.27 \\
\hline 0.2012 & 0.32 & 0.76 & -0.76 & 0.34 & -3.54 \\
\hline 0.3016 & 0.32 & 0.90 & -1.04 & 0.34 & -1.97 \\
\hline 0.4018 & 0.34 & 1.01 & -1.15 & 0.37 & -0.52 \\
\hline 0.5019 & 0.34 & 1.04 & -1.15 & 0.36 & 0.76 \\
\hline 0.6018 & 0.30 & 0.98 & -1.07 & 0.32 & 1.88 \\
\hline 0.7016 & 0.37 & 0.97 & -0.79 & 0.39 & 2.97 \\
\hline 0.8012 & 1.06 & 1.51 & 0.22 & 1.07 & 4.52 \\
\hline 0.9007 & 0.54 & 0.80 & 0.09 & 0.55 & 4.77 \\
\hline 1.0000 & 0.00 & 0.00 & 0.00 & 0.00 & 4.86 \\
\hline
\end{tabular}


Table 6. Percentage deviation between experimental and theoretical values of velocities in anisaldehyde + $m$-xylene system at different temperatures

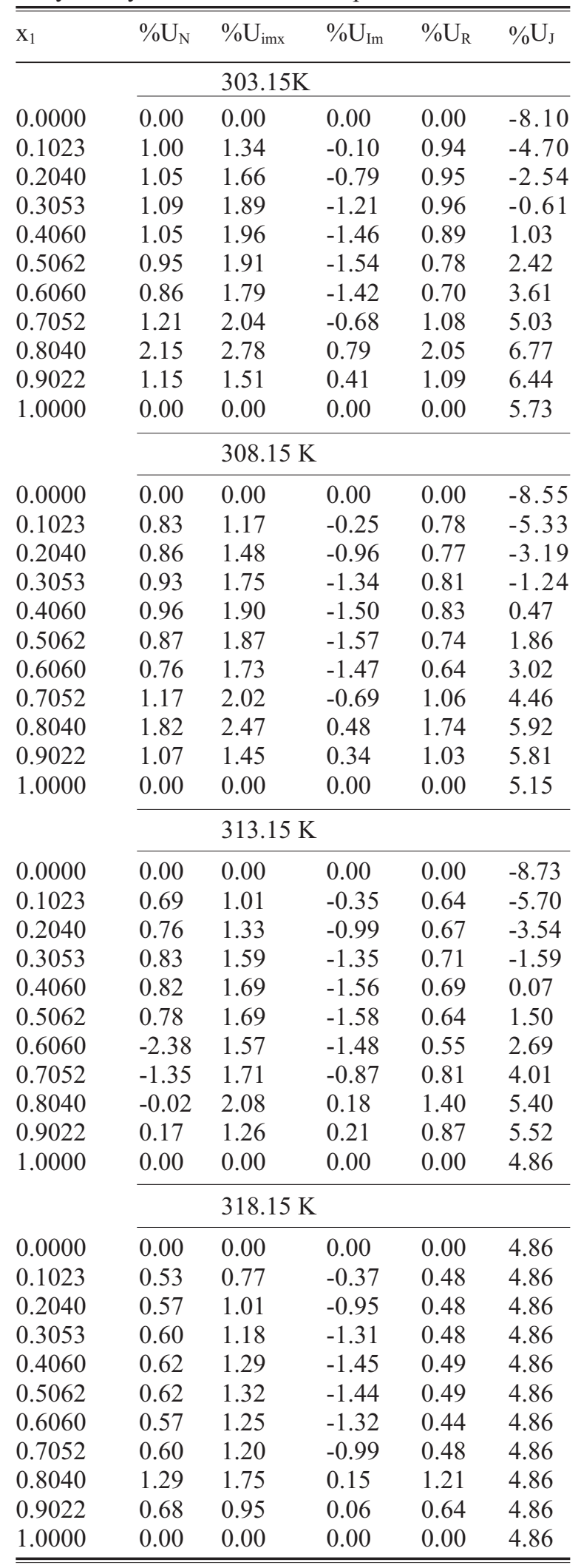

Table 7. Percentage deviation between experimental and theoretical values of velocities in anisaldehyde + $p$-xylene system at varying temperatures

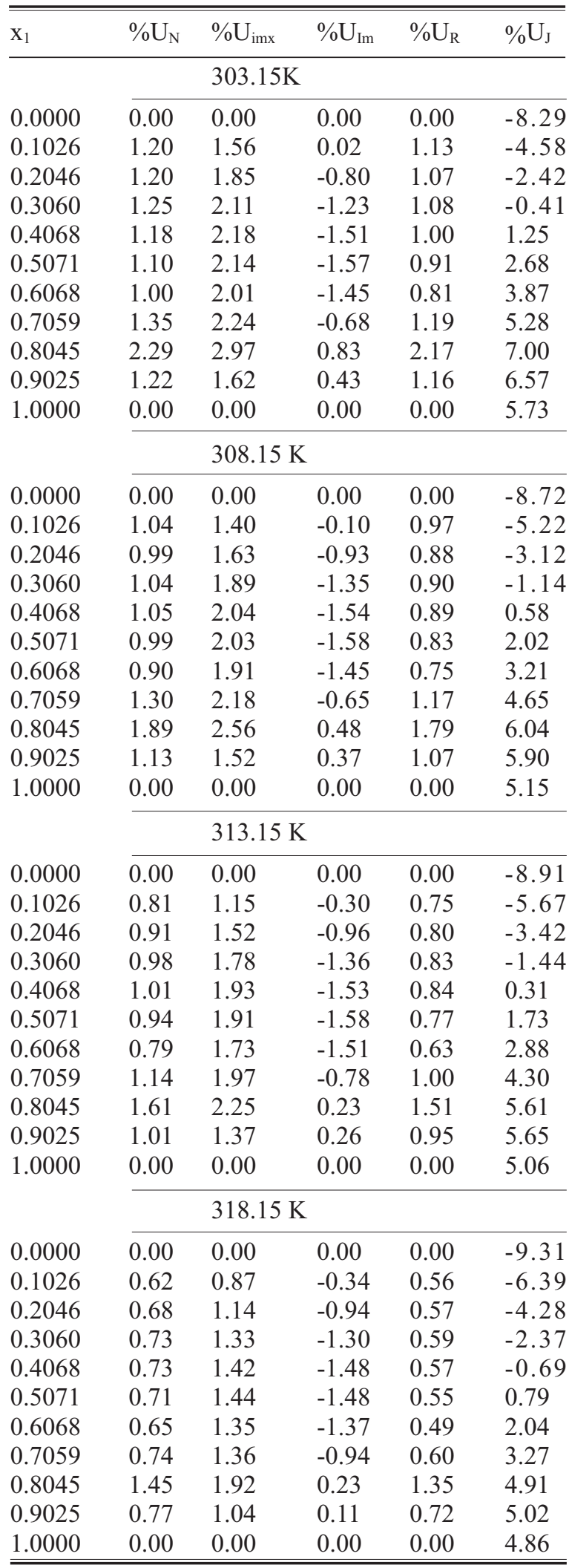




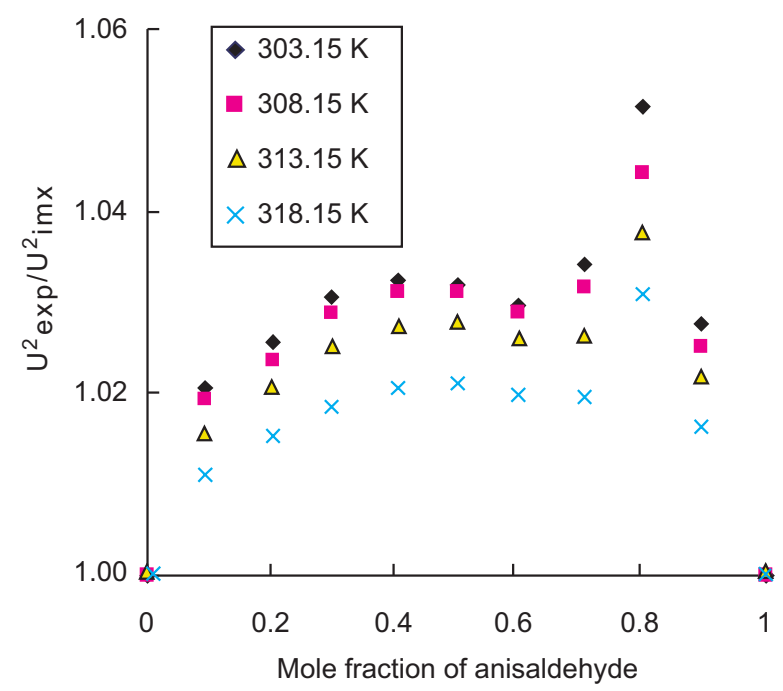

Fig. 1. Variation of $\mathrm{U}^{2}{ }_{\text {exp }} / \mathrm{U}^{2}{ }_{\text {imx }}$ in anisaldehyde $+o$-xylene system.

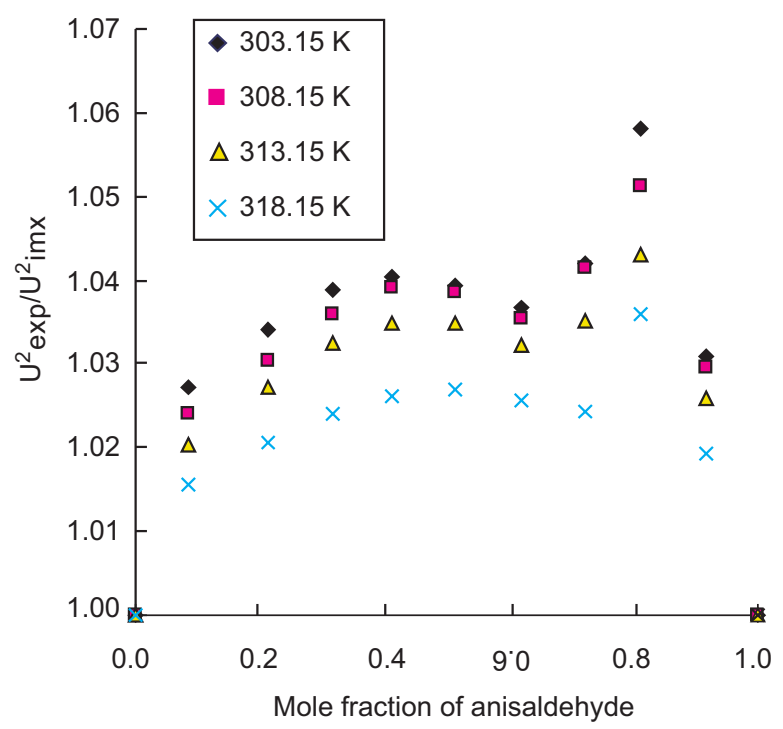

Fig. 2. Variation of $\mathrm{U}^{2}{ }_{\text {exp }} / \mathrm{U}^{2}{ }_{\mathrm{imx}}$ in anisaldehyde + $m$-xylene system.

to be maximum at mole fraction of anisaldehyde $=$ 0.8045 at all temperatures in all the three systems. The ratio $\mathrm{U}_{\text {exp }}^{2} / \mathrm{U}^{2}$ imx is used to measure the non-ideality in liquid mixtures, especially in those cases where the properties other than sound velocity are not known.

\section{Conclusion}

Theoretical evaluations of ultrasonic velocities in three binary liquid mixtures were determined, and the validity of different theories was checked. It was observed that

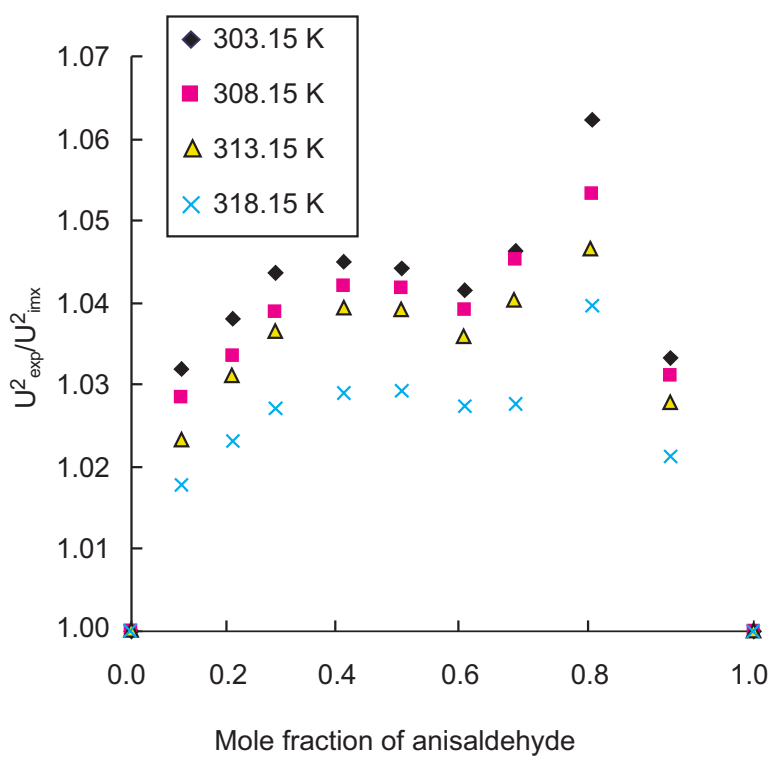

Fig. 3. Variation of $\mathrm{U}^{2}{ }_{\exp } / \mathrm{U}^{2}{ }_{\mathrm{imx}}$ in anisaldehyde $+p$-xylene system.

of all the theories Nomoto's theory gave the best results, followed by Rao's theory, in all the systems studied. Such conclusions are also derived by other researchers through their studies on different mixtures.

\section{References}

Ali, A., Yasmin, A., Nain, A.K. 2002. Study of intermolecular interactions in binary liquid mixtures through ultrasonic speed measurements. Indian Journal of Pure and Applied Physics, 40: 315-322.

Ali, A.A., Nain, A.K., Hyder, S. 2001. Study of intermolecular interaction in binary liquid mixtures through ultrasonic speed measurement. Journal of Pure and Applied Ultrasonics, 23: 73-79.

Al-Kandary, J.A., Al-Jimaz, A.S., Abdul-Latif, A.H.M. 2006. Viscosities, densities and speeds of sound of binary mixtures of benzene, toluene, $o$-xylene, $m$-xylene, $p$-xylene and mesitylene with anisole at 288.15. 293.15, 298.15 and 303.15 K. Journal of Chemical and Engineering Data, 51: 2074-2082.

Anuradha, S., Prema, S., Rajagopal, K. 2005. Ultrasonic studies on molecular interactions in binary mixtures of acetonitrile with carbonyl molecules. Journal of Pure and Applied Ultrasonics, 27: 49-54.

Arumugam, V., Sanjeevi, R., Raghunatha Rao, D., Shameem B. 1998. Classical areas of Pheno-menology (Including applications) - Ultrasonic studies on edible oils. Indian Journal of Pure and Applied Physics, 36: 578-583. 
Azhagiri, S., Jayakumar, S., Padmanaban, R., Gunasekaran, S., Srinivasan, S. 2009. Acoustic and thermodynamic properties of binary liquid mixtures of benzaldehyde in hexane and cyclohexane. Journal of Solution Chemistry, 38: 441-448.

Baluja, S., Parsania, P.H.1995. Acoustical properties of 3 -and alpha-furyl acrylic acid in protic and aprotic solvents. Asian Journal of Chemistry, 7: 417-423.

Baskaran, R., Kubendran, T.R. 2008. Thermophysical properties of para-anisaldehyde (1) + chlorobenzene (2) at temperatures of $(303.15,313.15$ and 323.15) $\mathrm{K}$ and a pressure of $0.1 \mathrm{Mpa}$. Journal of Chemical and Engineering Data, 53: 978-982.

Bhatia, S.C., Bhatia, R., Dubey, G.P. 2010. Thermophysical and sonochemical behaviour of binary mixtures of hexan-1-ol with halohydrocarbons at 303.15 K. Journal of Molecular Liquids, 152: 39-52.

Kumar, D.S., Rao, D.K. 2007. Study of molecular interactions and ultrasonic velocity in mixtures of some alkanols with aqueous propylene glycol. Indian Journal of Pure and Applied Physics, 45: 210-220.

Lide, D.R. (ed) 1995. CRC Handbook of Chemistry and Physics. $76^{\text {th }}$ edition, CRC Press, Boca Raton, FL, USA.

Narendra, K., Narayanamurthy, P., Srinivasu, Ch. 2010. Experimental and theoretical evaluation of ultrasonic velocities in binary liquid mixture cyclohexane $+o$-xylene at 303.15, 308.15, 313.15 and $318.15 \mathrm{~K}$. International Journal of Computational Mathematical Ideas, 2: 55-59.

Nomoto, O. 1958. Empirical formula for sound velocity in liquid mixtures. Journal of the Physical Society of Japan, 13: 1528-1532.

Perrin, D.R. 1980. Purification of Laboratory Chemicals, $2^{\text {nd }}$ edition, Pergamon Press, Oxford, UK.

Rao, G.V.R., Sri, P.B.S., Sarma, A.V., Rambabu, C. 2007. Comparative study of theoretical ultrasonic velocities of binary mixtures of methanol and pyridine at different temperatures. Indian Journal of Pure and Applied Physics, 45: 135-142.

Rao, G.V.R., Sarma, A.V., Krishna, J.S.R., Rambabu, C. 2005. Theoretical evaluation of ultrasonic velocities in binary liquid mixtures of $O$ chlorophenol at different temperatures. Indian Journal of Pure and Applied Physics, 43: 345-354.

Rastogi, M., Awasthi, A., Guptha, M., Shukla, J.P. 2002. Ultrasonic investigation of X-HO bond complexes. Indian Journal of Pure and Applied Physics, 40: 256-263.

Saravanakumar, K., Baskaran, Z.R., Kubendran, T.R. 2010. Acoustic and thermodynamic properties of binary liquid mixtures of acetophenone and benzene. Journal of Applied Science, 10: 1616-1621.

Uvarani, R., Punitha, S. 2009. Theoretical prediction of ultrasonic velocity in organic liquid mixtures. E- Journal of Chemistry, 6: S235-S238. 